\title{
ADI: un enfoque de aprendizaje en construcción en el contexto de la modalidad B-Learning ${ }^{1}$
}

\author{
Marbelys Elizabeth Cánchica de Medina ${ }^{2}$ \\ Universidad Nacional Experimental "Francisco De Miranda" (UNEFM), Venezuela
}

Recibido, abril 6 de 2015

Concepto evaluación, octubre 2 de 2015

Aceptado, octubre 15 de 2015
Referencia: Cánchica de Medina, M. (2015). “ADI: un enfoque de aprendizaje en construcción en el contexto de la modalidad B-Learning". Revista Academia y Virtualidad, 8, (2), 34-47

\section{Resumen}

Este breve artículo tiene como propósito socializar el conocimiento generado a partir una investigación de mayor alcance realizada por Cánchica (2014). La interpretación de las representaciones conceptuales sobre aprendizaje dialógico interactivo (ADI) - provisto por actores sociales de la modalidad B-Learning de la Universidad Nacional Experimental "Francisco de Miranda" (UNEFM) - se constituyó en uno de los objetivos específicos de estudio, el cual se desarrolló desde una perspectiva cualitativa y aplicó procedimientos metodológicos del estudio de casos y la teoría fundamentada, facilitando así el proceso de codificación y el análisis de la información recolectada.

Los resultados develan la emergencia de cuatro categorías principales; sin embargo, en esta oportunidad sólo se presenta la discusión y conclusión provisoria de uno de los hallazgos: "ADI es un enfoque pedagógico en construcción"; los actores sociales de la UNEFM manifiestan la necesidad de apropiarse del uso de las tecnologías de la información y comunicación (TIC) en la modalidad B-Learning, siguiendo un conjunto de principios propios de un modelo comunicativo no bancario donde el rol principal del docente es la de un agente innovador.

Palabras clave: B-Learning, innovación educativa, teoría fundamentada, aprendizaje dialógico interactivo, actores sociales, enfoque pedagógico.

\section{IDL: A learning approach under process within a B-Learning scenery}

\begin{abstract}
This short article aims at socializing the knowledge from a far-reaching research by Cánchica (2014). The interpretation of conceptual representations upon dialogic principles of interactive learning (IDL) provided by social stakeholders of B-Learning at Universidad Nacional Experimental "Francisco de Miranda" (UNEFM), became one of the specific

1. Artículo de investigación.

2. Doctora en Innovaciones Educativas. Docente titular Universidad Nacional Experimental "Francisco de Miranda". Adscrita al Departamento de Tecnología Educativa, Falcón, Venezuela 4101.Correo: marbelysc@gmail.com
\end{abstract}


objectives of this study developed from a qualitative perspective and applied methodological procedures of case study and the grounded theory, thus facilitating encoding process and analysis of the information gathered. Results reveal the emergence of four major categories, however this time only offers a provisional discussion and conclusion: "IDL is a pedagogical approach in process"; the social stakeholders at UNEFM speak about the need to adapt the use of information and communication technologies (ICT) regarding B-Learning, following a set of typical principles of a non-banking, communicative model where the main role of the teacher is an innovative agent.

Keywords: B-Learning, educational innovation, grounded theory, dialogic interactive learning, social stakeholders, pedagogical approach.

\section{ADI: uma abordagem de aprendizado em construção no quadro da modalidade B-Learning}

\section{Resumo}

Este breve artigo tem como propósito a socialização do conhecimento gerado a partir duma pesquisa de maior alcance realizada por Cánchica (2014). A interpretação das representações conceptuais sobre aprendizado dialógico interativo (ADI) - provido por atores sociais da modalidade B-Learning da Universidade Nacional Experimental "Francisco de Miranda" (UNEFM)- constituiu-se num dos objetivos específicos do estudo, o qual desenvolveu-se desde uma perspectiva qualitativa e aplicou procedimentos metodológicos do estudo de caso e a teoria fundamentada, facilitando assim o processo de codificação e análise da informação coletada. Os resultados revelam a emergência de quatro categorias principais, porém nesta oportunidade só se apresenta a discussão e conclusão provisória dum dos achados. "ADI é uma abordagem pedagógica em construção"; os atores sociais da UNEFM manifestam a necessidade de se apropriar do uso das tecnologias de informação e comunicação (TIC) na modalidade B-Learning, seguindo um conjunto de princípios próprios dum modelo comunicativo não bancário onde o role principal do docente é o de um agente inovador.

Palavras chave: B-Learning, inovação educativa, teoria fundamentada, aprendizado dialógico interativo, atores sociais, abordagem pedagógica.

\section{Introducción}

La Universidad Nacional Experimental "Francisco de Miranda" (UNEFM), ubicada en Santa Ana de Coro, estado Falcón (Venezuela), elaboró el proyecto Estudios Dirigidos (EDI) en 2002, basado en la necesidad de implementar una modalidad flexible y paralela a la presencial, de tal modo que permitiera albergar y profesionalizar en esta casa de estudio, a personas que por distintas limitaciones no ingresaban al sistema tradicional (Coello, Hernández \& Subero, 2002).
Más adelante, EDI pasa a denominarse aprendizaje dialógico interactivo (ADI), manteniendo en esencia los estudios semipresenciales (Reglamento EDI/ADI, 2002), llamados en el ámbito de la tecnología educativa estudios B-Learning, dado que combinan e integran sesiones de aprendizaje cara a cara con encuentros virtuales durante el proceso de formación (Torres, 2014 y Turpo, 2012).

No obstante, la idea impulsora de la investigación obedece a que el significado de ADI es confuso una vez que se analizan los documentos institucionales que lo refieren: 
ADI: un enfoque de aprendizaje en construcción en el contexto de la modalidad B-Learning

Reglamento EDI/ADI (2002); “justificación del cambio del nombre Estudios Dirigidos" (Coello \& Perozo, 2005); y, el libro Aprendizaje Dialógico Interactivo, una herramienta para el logro de una educación para la libertad (Talavera \& Yépez, 2009). La carencia de una ontología en cuanto a su significado parece indiscutible, percepción que se refuerza con las apreciaciones expuestas por un continuo de sujetos en el marco del desarrollo de sus experiencias docentes a nivel de pregrado, postgrado y formación continua (Pernalete, Cánchica \& Díaz, 2008 y Cánchica, 2009).

Ahorabien, siADI sehaejecutadodemaneraininterrumpida desde 2002, a pesar del nudo crítico identificado, entonces indiscutiblemente se cuenta con un conjunto de actores sociales innovadores que han ido adoptando, recreando o construyendo este concepto de manera individual y/o colectiva, que requiere ser develado, como insumo para la renovación y actualización congruente de su ontología institucional. Con base en los argumentos expuestos, se planteó como uno de los objetivos de la investigación,

"[...] interpretar las representaciones conceptuales sobre Aprendizaje Dialógico Interactivo (ADI) provisto por actores sociales de la modalidad BLearning de la Universidad Nacional Experimental 'Francisco de Miranda' (UNEFM)'.

En este sentido, se han recogido un cúmulo de vivencias de docentes catalogados por sus pares como innovadores que dan cuenta de la existencia de variadas lecturas que pueden agruparse básicamente en una dimensión subjetiva e interpretativa. Las categorías emergentes de la investigación, han declarado que: a) ADI en teoría es el B-Learning de la UNEFM; b) ADI en la práctica es una manifestación de constantes cambios; c) ADI simbólicamente es un segundo hogar; y d) ADI, desde lo pedagógico, representa un enfoque de aprendizaje en construcción.

En el marco del presente artículo, sólo se abordará el análisis realizado y las conclusiones provisorias del último constructo teórico develado.

\section{Metodología}

A partir de la premisa que la realidad social se construye con base en las interpretaciones que los actores le asignan a ésta, se ha asumido una postura socioconstruccionista que ha cobrado sentido al reconocer que el contexto abordado manifiesta un carácter dinámico que se construye y reconstruye desde los sujetos implicados, fundamentado precisamente en la capacidad individual y colectiva que surge de sus procesos de interacción.

En este sentido, asumir una perspectiva cualitativa con énfasis en lo interpretativo, ha permitido interpretar e indagar sobre una realidad y su entorno físico y social, permitiendo descubrir cómo suceden los hechos, cómo interactúan los actores sociales, y cuál es la dimensión de los cambios que en ella se producen (Goetz, \& Le Compte, 1988). Vale señalar asimismo que el estudio tuvo lugar en el entorno natural de los actores involucrados, modalidad B-Learning de la Universidad Nacional Experimental "Francisco de Miranda" (UNEFM), prestándole especial atención a los significados asociados al concepto de aprendizaje dialógico interactivo .

Siguiendo a Coello (2010), las fases de la investigación fueron:

a. la selección y definición de la situación problemática

b. planificación de la investigación

c. recolección sistemática de información

d. selección y descripción del caso

e. análisis, interpretación y verificación de la información

f. sistematización y publicación de hallazgos

Es importante igualmente justificar que la selección del método seleccionado, teoría de casos y teoría fundamentada, obedece a que su aplicación desde una epistemología introspectiva vivencial permite abordar distintos niveles de abstracción durante el estudio, para 
transitar desde un nivel descriptivo, interpretativo, iluminativo o de revelación y explicativo, de tal modo que pudiera incluso llevar finalmente hacia la predicción.

Por su parte, la selección de los actores sociales estuvo sujeta a un muestreo teórico de acuerdo con determinados criterios. Con base en el criterio de intención y pertinencia, fue preciso considerar en primer lugar, a los docentes que laboraban en la modalidad B-Learning de la UNEFM desde los inicios de su implementación hasta la fecha en que se hacía la investigación y, que además, fuesen calificados por sus pares como innovadores, siendo que sus discursos aportarían significados relevantes en relación con los cambios e innovaciones en las que habían participado o construido en los últimos diez años.

En segundo lugar, se tomaron casos adicionales, los cuales fueron escogidos en la medida en que el proceso de análisis de la información requería refinamiento y expansión de nuevas interpretaciones de las ya alcanzadas. Cuando las entrevistas no condujeron a nuevas comprensiones, en ese momento la totalidad de los informantes fue definida, a los fines de alcanzar el punto de saturación teórica (Strauss $\&$ Corbin, 2002). Siendo así, se lograron aplicar un total de 10 entrevistas a profundidad, diseminadas entre diez docentes (D1 a D10) que fueron complementadas con la participación de los tres autores del proyecto inicial de la modalidad B-Learning de la UNEFM (AUT1 a AUT3). Por tanto, la totalidad de los sujetos entrevistados fue de 13.

El contenido de las entrevistas abordó como elemento central vivencias, ideas, creencias, sentimientos, discursos $\mathrm{y}$ acciones de los actores, en cuanto el significado del ADI-UNEFM. La información obtenida se sirvió, según Cánchica (2014), de herramientas analíticas para aplicar los procesos de codificación respectivos, a saber:

a) Codificación abierta: proceso analítico por medio del cual se identifican los conceptos y se descubren en los datos sus propiedades y dimensiones.

b) Codificación axial: proceso de relacionar las categorías a sus subcategorías, denominado axial por- que la codificación ocurre alrededor del eje de una categoría, y enlaza el resto desde sus propiedades y dimensiones.

c) Codificación selectiva: proceso para integrar y refinar la teoría.

Por consiguiente, en este proceso específico se siguieron cuatro etapas fundamentales: la primera implicó la comparación de los datos; la segunda dio paso a la integración de cada categoría con sus propiedades; la tercera requirió delimitar la teoría que comenzaba a desarrollarse; y por último, se llegó a la cuarta etapa tras un proceso de saturación de los incidentes pertenecientes a cada categoría, permitiendo en atención a las aseveraciones formuladas redactar la teoría sustantiva emergente.

\section{Resultados y discusión}

A continuación se presentan los resultados y el análisis realizado en la investigación. El procedimiento seguido recoge una muestra teórica de expresiones con base en las inquietudes y propósitos del estudio, a los cuales se le ha realizado un proceso de codificación que ha permitido finalmente develar las categorías emergentes en cuanto a las interpretaciones conceptuales sobre el ADI-UNEFM.

En este orden de ideas, vale citar una de las aseveraciones (código in vivo) centrales, realizadas por un docente del área de educación de la modalidad B-Learning de la UNEFM, quien al preguntársele sobre qué significaba para éste el aprendizaje dialógico interactivo, refirió textualmente lo siguiente:

Lo dialógico interactivo es el enfoque que se le da a la modalidad, aunque cualquier institución puede tenerla, nosotros la aplicamos en una modalidad mixta cuya nomenclatura internacional se llama BLearning (AUT4).

La presente perspectiva sobre ADI, resulta una de las contribuciones más interesantes de los hallazgos que han sido develados en la totalidad de la investigación, considerando que aun cuando el término tiene sus raíces 
en antecedentes pedagógicos propuestos originalmente por Freire (2002), institucionalmente sólo se ha declarado que ADI es un enfoque, en el documento correspondiente al Programa de Formación Docente en Estrategias Didácticas con TIC (FEDITIC) expuesto por Coello, Peña \& Pernalete (2008).

La emergencia de aseveraciones similares a las presentadas también fueron identificadas en testimonios de otros actores sociales entrevistados:

... Más que cambiar programas, ADI se centra en cambiar la forma como vemos el aprendizaje...es un cambio de paradigma, en el cómo concebir los procesos de enseñanza y aprendizaje... paradigma diferente, modalidad diferente (D3).

Es decir, la orientación pedagógica de los procesos de enseñanza y aprendizaje que se desarrollan en la modalidad B-Learning de la UNEFM suponen una diferenciada manera de ser abordados, lo cual apunta a la comprensión de un concepto sobre cómo aplicar lo dialógico y lo interactivo en estos entornos. Pero precisamente allí está el dilema, pues en líneas preliminares ya habíamos referido que en la UNEFM se carece de una ontología institucional sobre el significado de ADI, lo cual ha sido confirmado por sus actores sociales, tal como se evidencia en el siguiente testimonio:

... Sin embargo en términos prácticos todavía nosotros no hemos consolidado lo que significa la modalidad o el enfoque que queremos, siento que la debilidad es producto de la misma cotnfusión. Pero como estamos tratando de innovar, dentro del caos hemos ido errando, pero poco a poco también nos estamos aproximando más hacia una definición más clara de lo que debemos hacer pedagógicamente en la modalidad (D4).
Siendo así, se reconoce que la misma dinámica de la modalidad B-Learning de la UNEFM ha reconocido la existencia de problemas en cuanto al significado institucional de ADI, pero se interpreta que a su vez, son los mismos actores sociales quienes lo han ido deconstruyendo y construyendo en la misma práctica que realizan. De allí que parezca oportuno develar los atributos de este concepto que han sido manifiestos por los entrevistados:

\section{Categorías asociadas al concepto. ADI como enfoque de aprendizaje de la modalidad B- Learning de la UNEFM}

Para este apartado se ha tomado una muestra de incidentes expuestos por los actores sociales que pone de manifiesto los atributos del aprendizaje dialógico interactivo como enfoque de la modalidad B-Learning de la UNEFM.

\section{Aprendizaje mediado por un diálogo constante}

De este modo, se puede interpretar que ciertamente el diálogo es una modalidad de comunicación, que se puede utilizar como instrumento para facilitar contenidos que propicien aprendizajes, a través de la interacción y de las TIC. La recomendación didáctica es que se realice de manera continua durante todas las fases del proceso de instrucción. En este orden de ideas, se tiene que para Freire (1997) la dialogicidad es una condición indispensable para el conocimiento, lo que implica una postura crítica y una preocupación por aprehender los razonamientos que median entre los actores, con el cual se promueve la construcción del conocimiento.

De acuerdo con ello, se asume que el aprendizaje dialógico interactivo como postura pedagógica demanda la aplicación de estrategias comunicativas que medien los aprendizajes. Con base en estas aseveraciones, se induce una vez más que los actores sociales asumen el ADI desde una perspectiva formativa, con sentido pedagógico. 
Marbelys Elizabeth Cánchica de Medina

\begin{tabular}{|c|c|c|}
\hline Muestra de incidentes & Subcategorías & Categoría \\
\hline $\begin{array}{l}\text { "ADI se basa en un } \\
\text { aprendizaje con diálogo" } \\
\text { (D2). }\end{array}$ & \multirow[t]{2}{*}{ Aprendizaje con diálogo } & \multirow{4}{*}{ Diálogo didáctico } \\
\hline $\begin{array}{l}\text { "El aprendizaje va basado } \\
\text { en un diálogo" (D5). }\end{array}$ & & \\
\hline $\begin{array}{l}\text { "A través del diálogo se } \\
\text { desarrollan contenidos de } \\
\text { aprendizaje" (D11). }\end{array}$ & Contenidos con diálogos & \\
\hline $\begin{array}{l}\text { "El diálogo es una forma } \\
\text { de comunicar contenidos } \\
\text { de aprendizaje" (D10). }\end{array}$ & $\begin{array}{l}\text { Comunicación para los } \\
\text { aprendizajes }\end{array}$ & \\
\hline $\begin{array}{l}\text { "En el enfoque dialógico } \\
\text { interactivo debe haber una } \\
\text { constante comunicación" } \\
\text { (D3). }\end{array}$ & Constante comunicación & Constancia en el diálogo \\
\hline
\end{tabular}

Tabla 1. Categorías y subcategorías del aprendizaje mediado por el diálogo constante

\begin{tabular}{|c|c|c|}
\hline Muestra de incidentes & Atributos & Subcategorías \\
\hline $\begin{array}{l}\text { “...El diálogo es } \\
\text { multidimensional. } \underline{\text { La }} \\
\text { interacción puede darse } \\
\text { de los estudiantes entre sí, } \\
\underline{\text { consigo mismos, con el }} \\
\text { docente, con la realidad, con } \\
\text { las actividades y materiales } \\
\text { de aprendizaje" (AUT 4). }\end{array}$ & $\begin{array}{l}\text { Estudiantes entre sí. } \\
\text { Estudiantes consigo } \\
\text { mismos } \\
\text { Estudiantes con el docente } \\
\text { Con su contexto. } \\
\text { Con actividades } \\
\text { Con materiales de } \\
\text { aprendizaje, }\end{array}$ & $\begin{array}{l}\text { Diálogo en diferentes } \\
\text { direcciones }\end{array}$ \\
\hline $\begin{array}{l}\text { "...teniendo claro que el } \\
\text { crecimiento intelectual } \\
\text { no solo viene dado por } \\
\text { los recursos que son } \\
\text { administrados por el } \\
\text { profesor, sino de otras } \\
\text { personas que pueden tener } \\
\text { influencia en la formación } \\
\text { del alumno" (D8). }\end{array}$ & $\begin{array}{l}\text { Personas externas al aula } \\
\text { de clases. }\end{array}$ & $\begin{array}{l}\text { Diálogo con diferentes } \\
\text { agentes }\end{array}$ \\
\hline $\begin{array}{l}\text { “...Es más dentro del mismo } \\
\text { diálogo podemos concebir } \\
\text { la parte dialógica dentro de } \\
\text { lo que es el aula virtual” } \\
\text { (AUT 4). }\end{array}$ & Aula virtual. & Diálogo virtual. \\
\hline $\begin{array}{l}\text { “...Diálogo de saberes entre } \\
\text { estudiantes, el docente y la } \\
\text { comunidad a través de una } \\
\text { plataforma”(D7). }\end{array}$ & Plataforma. & \\
\hline $\begin{array}{l}\text { “...Lo dialógico yo lo aplico } \\
\text { más en la presencialidad, } \\
\text { pero también lo puedo hacer } \\
\text { con el foro donde quedan los } \\
\text { registros" (D8). }\end{array}$ & Presencialidad. & Diálogo presencial. \\
\hline
\end{tabular}

Tabla 2. Atributos y subcategorías del diálogo multidimensional

\section{Diálogo multidimensional}

El rasgo que se ha denominado diálogo multidimensional, se justifica precisamente por el conjunto de dimensiones que lo conforman; a saber: agentes que interaccionan, direccionalidad con la cual se comunican, y el escenario donde se desarrolla, tal como se evidencia en la tabla siguiente:

En este sentido, se tiene que el proceso dialógico puede ocurrir entre actores sociales que participan tradicionalmente en el hecho educativo (alumno y docente), pero también se prevé la posibilidad que los estudiantes interaccionen entre sí, con los materiales educativos, con sus tareas, y consigo mismos cuando piensan, reflexionan, cuando aplican el pensamiento crítico, cuando realizan procesos metacognitivos, entre otros. Todos estas formas de interacción ya habían sido estudiadas por teorías de la educación a distancia citadas por García (2001); sin embargo, representa una novedad asumir que desde una modalidad B-Learning se le dé lugar al contexto, llámese comunidad, la familia, los expertos, como nuevos aliados de la intervención didáctica.

Por otra parte, resulta importante indicar que tales agentes pueden participar tanto en escenarios físicos o virtuales, donde el flujo de la comunicación no sólo se desarrollará de uno a uno, por ejemplo docente-alumno, sino que integrará más sujetos sumándolos al proceso de discusión o debate, entre otras actividades que puedan plantearse, a los fines que se abra paso a diálogos que se ejecuten en ambos o en diversos y variados sentidos.

De este modo se concibe que el aprendizaje dialógico interactivo tenga como otro de sus principios la aplicación de un diálogo multidimensional en atención a los agentes que interactúan, el flujo o direccionalidad de la comunicación, y los escenarios donde se realiza; atributos que representan un conjunto de opciones posibles para diseñar estrategias de aprendizajes en el marco de la modalidad B-Learning. 
ADI: un enfoque de aprendizaje en construcción en el contexto de la modalidad B-Learning

\section{Diálogo simétrico}

La categoría denominada simetría se justifica por los atributos que los actores sociales le otorgan a las condiciones del diálogo, a saber: en un mismo nivel, comunicación horizontal, sin jerarquías.

\begin{tabular}{|c|c|c|}
\hline Muestra de incidentes & Atributos & Subcategorías \\
\hline $\begin{array}{l}\text { “...Es más rico y más } \\
\text { divertido cuando el } \\
\text { conocimiento dentro del } \\
\frac{\text { aula se da dentro de un }}{\text { mismo nivel...”(D2). }}\end{array}$ & & \\
\hline $\begin{array}{l}\text { “...escuchamos más a los } \\
\text { estudiantes o conversamos } \\
\text { con ellos o aprendemos } \\
\text { de ellos, porque el único } \\
\text { que sabe no es el profesor, } \\
\text { ellos también tienen un } \\
\text { cuerpo de conocimientos” } \\
\text { (D8). }\end{array}$ & $\begin{array}{l}\text { Estudiantes tienen } \\
\text { también conocimientos }\end{array}$ & En un mismo nivel. \\
\hline $\begin{array}{l}\text { “...lo dialógico... debe } \\
\text { ser una comunicación } \\
\text { totalmente horizontal } \\
\text { en la cual no haya y no } \\
\text { se crea que se tiene más } \\
\text { conocimiento que el otro" } \\
\text { (AUT1). }\end{array}$ & $\begin{array}{l}\text { No se crea que el otro } \\
\text { tiene más conocimiento } \\
\text { que el otro }\end{array}$ & Sin jerarquías \\
\hline $\begin{array}{l}\text { "Diálogo de saberes" } \\
\text { (D2), (D7). }\end{array}$ & Diálogo de saberes & Diálogo de saberes \\
\hline
\end{tabular}

Tabla 3. Atributos y subcategorías del diálogo simétrico

La representación conceptual analizada concibe que en el proceso pedagógico se realice un acto del reconocimiento del otro, en el marco de las capacidades tácitas o académicas que posean los actores sociales involucrados. En este sentido es posible que se compartan saberes científicos como saberes populares en condiciones simétricas de horizontalidad, sin jerarquías, los cuales pueden interactuar entre sí para construir un nuevo conocimiento. Este hecho se fundamenta en las corrientes humanistas de la educación y apuestan a la posibilidad de enajenar al sujeto, o emanciparlo (Freire, 2002).

Siendo así, quienes estimen aplicar el aprendizaje dialógico interactivo como enfoque pedagógico, deben ser conscientes de las diferencias de los actores sociales (cultura, religión, raza, estatus académico, entre otros) sin subestimar sus saberes, a los fines de facilitar las mismas oportunidades y condiciones que den paso a la simetría necesaria. Desde esta perspectiva, la capacidad de contribuir con el conocimiento que se construye se aleja de procesos que controlan el pensamiento de los estudiantes y limitan su creatividad y criticidad, y ello es posible implementarlo en el aula, a través de las distintas formas de comunicación y en el intercambio de saberes.

\section{Comunicación mediada e interactiva}

Existe una polémica con relación a la redundancia del término dialógico e interactividad; no obstante, los sujetos entrevistados han presentado sus significados:

...pero generalmente la productividad de esa parte dialógica la hacemos más en el componente presencial con cada uno de los profesores ... por lo menos yo veo que es más fácil el registro o que se pueda comprobar la parte interactiva a través del mismo salón MOODLE (D8).

En este sentido, se interpreta que el componente dialógico de la modalidad se asocia a una forma de comunicación que se establece entre los individuos y que esencialmente sucede en los encuentros presenciales. Cuando se utilizan los medios tecnológicos en procesos comunicativos se abre paso a la interactividad, al igual que lo que sucede con un diálogo mediado (interacción). Esta teoría sustantiva coincide con lo planteado por Wagner:

[...] la interactividad parece haber emergido de descripciones de la capacidad tecnológica para establecer conexiones de punto al punto. . . en tiempo real. Así, la interacción se centra en comportamientos de la gente, mientras que la interactividad se centra en las características de los sistemas de la tecnología (1994).

En consecuencia, para que este proceso sea posible se requiere hacer uso de las TIC y promover experiencias donde pueda fluir el proceso comunicativo en las direcciones que convenga. En el marco de este análisis 
es favorable exponer otra muestra de incidentes que han dado lugar a explicaciones sobre la interactividad que se hace posible desde el ADI.

Por consiguiente, el ADI apuesta al uso de las TIC, especialmente las provenientes de la web 2.0 (blogs, wikis, entre otros), pues se constituyen en herramientas que potencian el diálogo social tanto sincrónico como asincrónico, además permiten proveer mensajes verbales, icónicos, pictóricos o sonoros que pueden ser elaborados en el marco de aprendizajes colaborativos.

Por otra parte, en la actualidad se dispone de distintas plataformas tecnológicas a través de las cuales se generan ambientes virtuales de aprendizaje, las cuales permiten además aplicar principios comunicativos en su diseño y apropiarse de las herramientas que proveen la interactividad para desafiar al estudiante, motivarlo, entretenerlo, estimular el descubrimiento y adaptarlo a los distintos ritmos y niveles del aprendizaje; en fin, propiciar aprendizajes socializados pero centrados en el estudiante. Los niveles de interactividad en estos cursos definitivamente seguirán siendo un reto para proveer aprendizajes de inmersión de alto nivel.
Bajo este principio los contactos cara a cara y la comunicación oral representan la opción más utilizada en el plano de la presencialidad; no obstante, la evolución tecnológica también lo posibilita de forma mediada con la adición de una amplia gama de opciones que permiten un nuevo lenguaje de uso frecuente, especialmente en el caso de los teléfonos inteligentes.

En este sentido, la comunicación mediada e interactiva se convierte en un principio ineludible del aprendizaje dialógico interactivo, como elemento que se abre a la posibilidad de aprovechar la potencialidad comunicativa que ofrecen las TIC.

\section{Inteligencia cultural colaborativa e intersubjetiva}

La oportunidad de aprender de otros y con otros, fue expresada por los actores sociales entrevistados. Por lo tanto, se interpreta que para que esto sea posible, existe un conjunto de condiciones que permitan tanto promoverla como afectarla.

\begin{tabular}{|c|c|c|}
\hline Muestra de incidentes & Atributos & Subcategorías \\
\hline $\begin{array}{l}\text { "...es como si el docente estuviera den- } \\
\text { tro del computador y le estuviera dando }\end{array}$ & Docente dentro del computador & \multirow{3}{*}{ Interactividad } \\
\hline $\begin{array}{l}\text { respuesta a esas preguntas o interrogan- } \\
\text { tes que tiene el alumno" (D2). }\end{array}$ & Retroalimentación electrónica & \\
\hline $\begin{array}{l}\text { "Estudiante Interactúa con herramien- } \\
\text { tas" (D2). }\end{array}$ & Relación estudiante-TIC & \\
\hline $\begin{array}{l}\text { “...Diálogo de saberes... a través de } \\
\text { una plataforma" (D7). }\end{array}$ & Diálogo a través de plataforma & Diálogo mediado \\
\hline "Emplea diversas herramientas" (D1). & \multirow[b]{2}{*}{ Diversidad de herramientas } & \multirow{4}{*}{ Demanda uso de las TIC } \\
\hline $\begin{array}{l}\text { "Usa herramientas que el profesor pone } \\
\text { a su disposición"(D5). }\end{array}$ & & \\
\hline "Se apoya en el uso de las TIC" (D4). & TIC & \\
\hline $\begin{array}{l}\text { "Uso de herramientas basada en la Web } \\
2.0 " \text { (D1). }\end{array}$ & Web 2.0 & \\
\hline
\end{tabular}

Tabla 4. Subcategorías y atributos de la comunicación mediada e interactiva 
ADI: un enfoque de aprendizaje en construcción en el contexto de la modalidad B-Learning

\begin{tabular}{|c|c|c|}
\hline Muestra de incidentes & Atributos & Códigos \\
\hline $\begin{array}{l}\text { "Es decir en ese aprendizaje to- } \\
\text { dos estamos aprendiendo del } \\
\text { otro, no es que el profesor sea } \\
\text { dueño del saber y el estudiante } \\
\text { no sabe nada. Todos tenemos un } \\
\text { nivel conocimientos y procedi- } \\
\text { mientos que facilitan al otro con } \\
\text { la interacción la posibilidad de su } \\
\text { aprendizaje" (A1) }\end{array}$ & $\begin{array}{l}\text { Todos aprendiendo del } \\
\text { otro }\end{array}$ & \multirow[t]{2}{*}{ Inteligencia cultural } \\
\hline $\begin{array}{l}\text { "... escuchamos más a los estu- } \\
\text { diantes o conversamos con ellos } \\
\text { o aprendemos de ellos, porque el } \\
\text { único que sabe no es el profesor, } \\
\text { ellos también tienen un cuerpo de } \\
\text { conocimientos." (D8) }\end{array}$ & Aprendemos de ellos & \\
\hline $\begin{array}{l}\text { "La idea de este enfoque es } \\
\text { que sea colaborativo y diverso } \\
\text { donde los estudiantes participen } \\
\text { libremente y estén más } \\
\text { involucrados en la construcción } \\
\text { de su propio aprendizaje" (D11). }\end{array}$ & $\begin{array}{l}\text { Colaborativo } \\
\text { Diverso } \\
\text { Participativo } \\
\text { Involucrados } \\
\text { Voluntario }\end{array}$ & $\begin{array}{l}\text { Construcción } \\
\text { colaborativa }\end{array}$ \\
\hline $\begin{array}{l}\text { "yo converso con ellos, creo } \\
\text { que eso es parte de lo dialógico, } \\
\text { escuchar la opinión de ellos al } \\
\text { respecto, tanto en... entonces } \\
\text { con base en eso } \underline{\text { legamos a }} \\
\text { un acuerdo en cada grupo es } \\
\underline{\text { diferente, independientemente }} \\
\text { que sea la misma unidad } \\
\text { curricular...ellos sugieren y yo } \\
\text { escucho" (D8). }\end{array}$ & $\begin{array}{l}\text { Conversar } \\
\text { Escuchar argumentos } \\
\text { Reflexiones y consensos } \\
\text { por grupos }\end{array}$ & Intersubjetividad \\
\hline
\end{tabular}

Tabla 5. Códigos y atributos del concepto "inteligencia cultural colaborativa intersubjetiva"

Se parte del reconocimiento que los sujetos que participan en el proceso educativo suelen tener personalidades, cualidades, conocimientos y posiblemente culturas diferenciadas, tal como se indicó cuando se estudió el principio de simetría del diálogo; no obstante, lo que sucede cuando aprovechamos estas diferencias puede generar ganancias al aprendizaje.

“...nosotros abarcamos o tomamos en cuenta lo que son la diversidad de los estudiantes que tenemos, su formas de aprendizaje, sus diferencias individuales y se le saca ventaja" (D3).
Esto significa que durante el proceso dialógico, conjuntamente, en pro de objetivos académicos o instruccionales específicamente, se encuentran e interactúan las diferencias de los distintos agentes que participan, incluyendo la diversidad de culturas. Este rasgo ha sido denominado por Freire (2002), como inteligencia cultural.

En el marco de los testimonios presentados en la tabla anterior, también figura el principio del aprendizaje colaborativo, el cual multiplica la posibilidad de abrirse a diferentes ideas, a la argumentación, a la responsabilidad social, competencias comunicativas, creatividad, capacidad cognitiva que permitan establecer nuevas rutas y estrategias para la solución de problemas comunes. Este componente del ADI se sustenta axiológicamente en el respeto a las ideas, la tolerancia de las diferencias y la solidaridad con quienes necesitan del otro.

En consecuencia, el aprendizaje desde esta perspectiva, alude también a la construcción del conocimiento que se hace posible desde la intersubjetividad, aplicable en procesos de colaboración con diversidad de agentes, el cual se vincula con procesos de análisis crítico, autorevisión de conceptos y comprensión socializada de significados.

Está visto entonces que la interacción entre los significados expuestos, permite ver como un todo la posibilidad de aplicar en el marco del enfoque los principios de inteligencia cultural con rasgos colaborativos e intersubjetivos.

\section{Rol dialógico del agente innovador}

El estudio ha reportado asimismo un conjunto de principios que deben orientar el aprendizaje dialógico interactivo en modalidad B-Learning, como en el caso de la UNEFM, pero también se han develado los roles que deben desempeñar los actores sociales vinculados al hecho educativo, especialmente el docente. 
Marbelys Elizabeth Cánchica de Medina

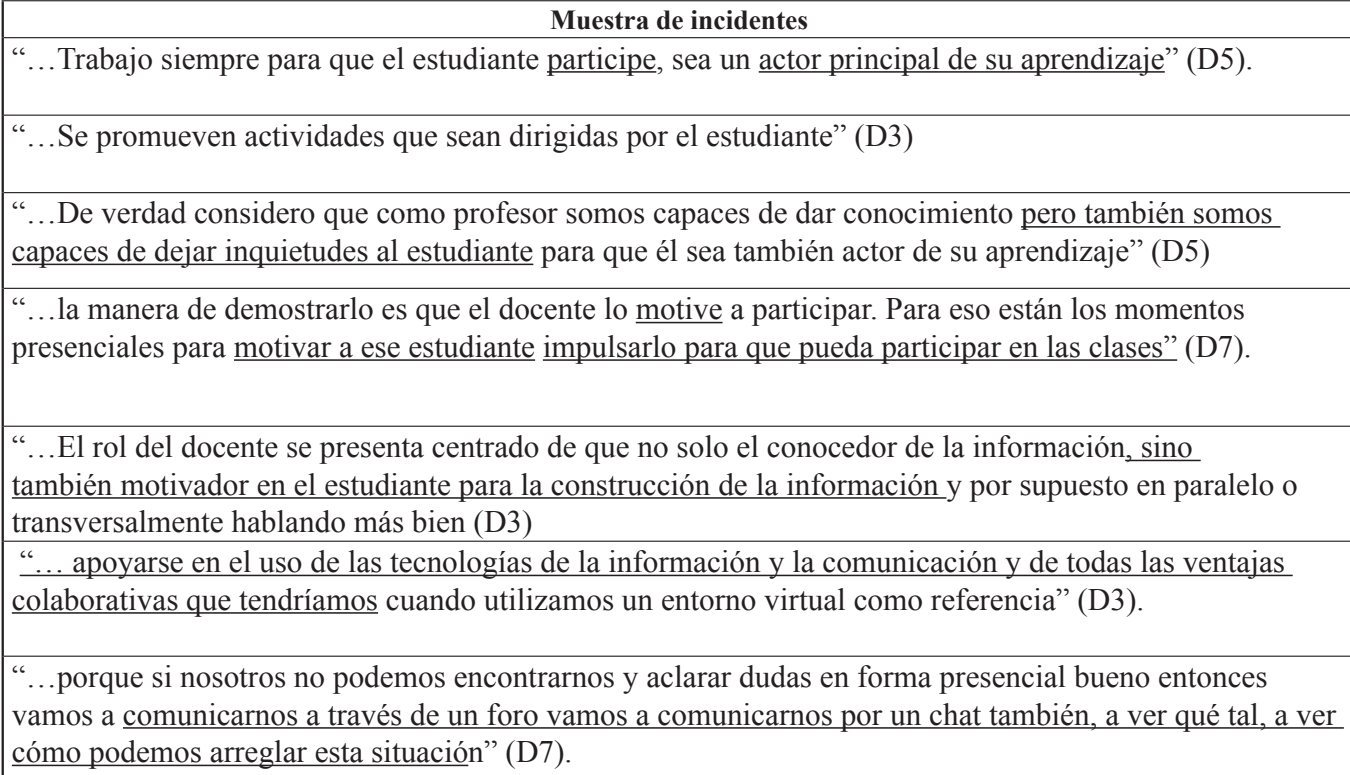

cómo podemos arreglar esta situación" (D7).

“...Se les deja información a los alumnos con un cronograma, se les envía mensajes constantemente ,se publican actividades, se monitorea lo que hacen...”(D1)

“...por supuesto no solo nos debemos limitar al uso de la plataforma en el caso de ADI, porque muchas veces, bueno la plataforma, no funcionó pero tienes un correo electrónico del profesor con el cual le puedes enviar la información o buscar otros medios utilizando los...recursos tecnológicos que no necesariamente tenga que ser la plataforma." (D6).

"...que los docentes sean más abiertos al uso de la modalidad ,al hecho que los docentes sean más activos al uso de la plataforma y sus opciones "(D3)

“...que el estudiante interactué en el foro y el profesor está al tanto de responder porque a veces nos centramos mucho en que exigimos a los estudiantes para que participen pero el profesor también debe dar esa participación" (D5).

“...el docente se debe esforzar un poquito más en crear materiales que tengan ese componente dialógico interactivo" (D6).

“...desarrollar en ellos la cultura de que las calificaciones se publican a través de ella o que los mensajes... los mensajes o circunstancias, novedades, informaciones que se pueden ir dando durante el desarrollo de

la actividad serán publicadas meramente tanto en el aula virtual como recursos externos adicionales como en el caso de grupos en línea" (D3).

"...Yo creo que un docente debe ser como un multi-idioma, las estrategias son idiomas para estimular al estudiante, el docente creo que no debería enseñar, tanto más bien debería estimularlo, promoverlo y estar atento a como el alumno aprende" (D4).

“...Ahora no, ahora simplemente los contenidos que se dan...contenidos básicos que sirvan de guía para la investigación para que lo use el estudiante y luego venga y lo analicemos, lo discutamos en el momento de la clase o bien sea a través de la red" (D1).

“...No es dador de clases" (D2, D5).

“...yo creo que la empatía es importante allí...” (D4).

“...evitando el vocabulario muy serio, o sea hacerlo un poco más cálido, más empático” (D1).

“...el aprendizaje puede desarrollarse más allá de las cuatros paredes del salón cuando así se requiere” (D5).

\begin{tabular}{|l|}
\multicolumn{1}{|c|}{ Atributos } \\
$\begin{array}{l}\text { Se orienta para que el } \\
\text { estudiante participe }\end{array}$ \\
$\begin{array}{l}\text { Plantea actividades para el } \\
\text { estudiante }\end{array}$
\end{tabular}

Despierta inquietudes

Motiva hacia la

participación

Motiva la construcción de la información

Usa las ventajas

de colaborativas y

comunicativas de las TIC

Usa distintos medios para

la comunicación.

Usas distintos medios para

proveer información y

monitorear al alumno

Utiliza recursos variados

además de la plataforma

virtual

Usa activamente la

plataforma virtual

Ofrece retroalimentación

oportuna

Crea materiales dialógicos.

Genera cultura de accesibilidad virtual

Aplica variedad de estrategias

Se analizan y discuten contenidos de aprendizajes

Usa Lenguaje empático

No se limita a proveer aprendizajes en el aula
Códigos

Facilitación del aprendizaje centrado en el estudiante

Gestión de aprendizaje dialógico con las TIC
Estimula el pensamiento crítico

Establece empatía con el estudiante Provee aprendizajes significativos

Tabla 6. Códigos asociados al rol dialógico que debe cumplir el docente en el ADI 
Evidentemente, los actores sociales del ADI-UNEFM han otorgado un conjunto de funciones a la acción docente para el B-Learning de la UNEFM, representando con ello una contribución importante para poner en práctica un aprendizaje dialógico interactivo como enfoque pedagógico innovador que rompe con esquemas tradicionales.

“...si el docente se cree el dueño de la información, del conocimiento y no le coloca desafíos al estudiante para que participe en la información, la busque, sea crítico, entonces el enfoque que no estará centrado en el alumno" (D3).

Por consiguiente, básicamente es importante identificar que el docente deberá asumir un compromiso con la facilitación y mediación de aprendizajes centrados en el alumno, a través del diálogo y la interactividad, generando condiciones para la implementación didáctica de un proceso que interconecta las dimensiones social, humana, afectiva y pedagógica, donde podrá ser acompañado por otros colaboradores que no necesariamente sean sus colegas, sino la comunidad, la familia, entre otros agentes.

A continuación, se resumen los códigos asociados al concepto develado a partir de la siguiente ilustración:

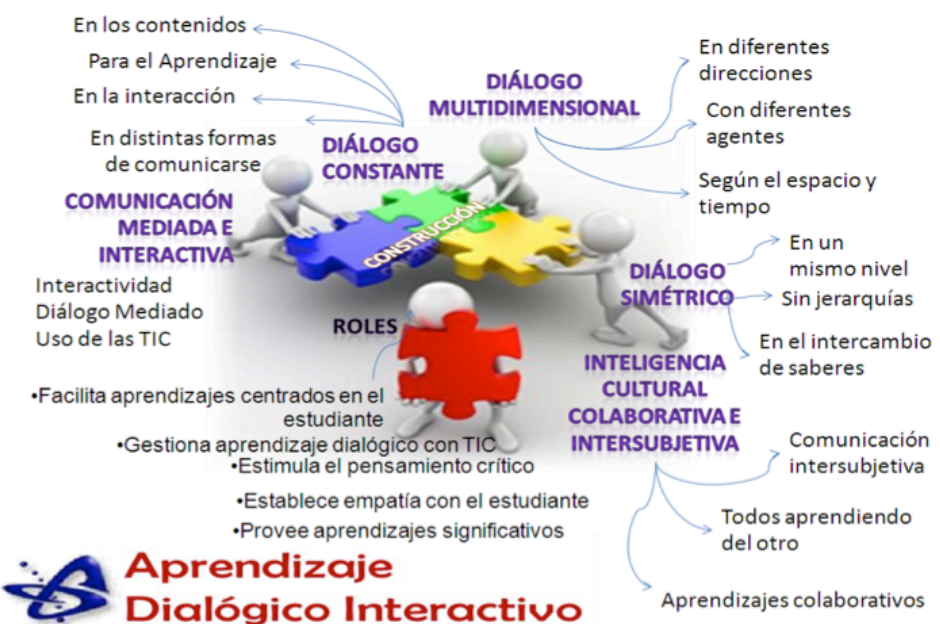

Figura 1. Síntesis de las representaciones del ADI como enfoque pedagógico
"ADI como enfoque de aprendizaje" es una representación conceptual emergente y construida por los actores sociales entrevistados, que apuesta a la aplicación de principios comunicativos emancipadores en los procesos instruccionales que se gestan en la modalidad B-Learning. En este sentido, refiere la necesidad de establecer diálogos desde los siguientes referentes: multidimensionalidad, simetría, constancia, comunicación mediada e interactiva, inteligencia cultural colaborativa e intersubjetiva, y la aplicación de roles por parte del agente de cambio, desglosados así:

a) El diálogo multidimensional se potencia cuando se realiza en diferentes direcciones, participan distintos tipos de agentes, y permite encuentros con opciones espacio-temporales, bien sea presencia o virtual.

b) El diálogo constante asume la necesidad de realizarlo de modo continuo como estrategia para el logro de los aprendizajes.

c) La comunicación mediada e interactiva se refiere a la apropiación didáctica de las TIC para potenciar pedagógicamente los procesos dialógicos entre hombre-máquina y entre sujetos

d) La simetría alude a la necesidad de establecer procesos comunicativos en un mismo nivel, donde se reconozca en el otro como par, y sean valoradas sus intervenciones.

e) La inteligencia cultural colaborativa e intersubjetiva, apuesta a contribuir los unos con los otros, en unión de esfuerzos para la construcción de conocimientos producto de la interacción y el intercambio, entre los actores sociales que participan.

Los principios declarados deben ser direccionados por el docente como facilitador y gestor en el uso de las TIC, a fin de promover el pensamiento crítico, el aprendizaje significativo y situaciones empáticas que favorezcan el proceso instruccional. 


\section{Conclusiones provisorias}

- La teoría sustantiva develada se puede transferir en el diseño de propuestas de innovación educativa que tiene lugar en los ambientes virtuales de aprendizaje y en el diseño propiamente de experiencias B-Learning.

- La dimensión tecnológica en la modalidad B-Learning, se convierte en una necesidad imperiosa cuando se reconoce que las tecnologías de información y de la comunicación (TIC) pueden servir a los objetivos estratégicos de la didáctica en el marco de la aplicación de los principios dialógicos develados. Se concibe entonces que el tratamiento de las herramientas tecnológicas considere rasgos semánticos y estructurales cualitativamente diferentes, en pro del aprovechamiento de la potencialidad comunicativa que ofrecen.

- El diálogo y la interactividad son principios que deben aplicarse en la generación de ambientes virtuales de aprendizaje (AVA), lo cual exige reinterpretar nuevas técnicas y procedimientos que superen las visiones instrumentales y pretendidamente neutras de una educación tradicional.

- El aprendizaje dialógico interactivo surge como alternativa que pretende superar las connotaciones negativas de la modalidad B-Learning; una propuesta que se ha gestado en el contexto de la UNEFM, fundamentada en principios de la innovación educativa y en el enfoque pedagógico comunicativo, con la finalidad de orientar estrategias de acción y sus consecuencias, desde la interacción de los componentes que deben conformar la modalidad B-Learning, a saber: rol de los actores, ambientes de aprendizaje, herramientas tecnológicas, materiales educativos, flexibilidad, comunicación, aprendizajes, y políticas nacionales e internacionales sobre democratización e inclusión de la educación.

- ADI como enfoque forma parte de un modelo comunicativo no bancario, constructivista y humanista, donde las relaciones didácticas entre docente, estudiantes, como también con la comunidad, se gestan de manera horizontal, estableciendo vías para el diálogo constante y estrategias para la formación de un ciudadano crítico reflexivo, que respeta y tolera la diversidad, pero argumenta y busca la conciliación de las partes.

- ADI como enfoque abre la posibilidad de permear tanto las actividades en construcción y procedimientos ligados al desarrollo del AVA, como los criterios para la planeación, diseño y ejecución de las actividades didácticas propiamente dichas de una modalidad B-Learning.

- Desde este enfoque de aprendizaje, el estudiante se considera el centro de la acción educativa, pero no en "soledad" sino en acompañamiento de sus pares y de personas que comparten (comunidad) sus diferentes grados de experticia en contextos de aprendizaje cooperativo y mediado. En este caso, el rol del docente es de facilitador-mediador que acompaña a sus estudiantes en el logro de sus metas pero sin imponer su visión o controlar su acción. Éste se constituye más bien en la persona que promueve el aprendizaje constructivista social dándole valor al diálogo para argumentar sus propuestas, someterlas a consideración y consolidar el consenso.

- El proceso que se desarrolla del ADI como enfoque se complementa del trabajo cooperativo, por ser un proceso dialéctico grupal, en el que todos los participantes realizan aportes a la solución de un problema y hacen uso compartido de la información, estableciendo acciones de consenso para la toma de decisiones, en un marco de pensamiento crítico, solidaridad, respeto, autonomía, autorregulación y democracia. En este sentido se promueve la interacción social, a través de la selección de los niveles de ayuda más apropiados, los cuales da a conocer a todos los participantes y determina la aparición y desaparición de dichas ayudas, en función de los avances en la tarea grupal (andamiaje).

- Se pretende que a partir del proceso del diseño de la instrucción, todos los participantes o responsables del proceso compartan y aprendan en comunidad, des- 
ADI: un enfoque de aprendizaje en construcción en el contexto de la modalidad B-Learning

de la interacción sociocultural y sometan a la crítica reflexiva sus producciones, propuestas, actividades, entre otros. Estableciendo vías para la comunicación permanente y estrategias que les permitan lograr los acuerdos sobre el proceso de desarrollo.

- La enajenación del sujeto y la transformación del individuo desde una pedagogía emancipadora sigue siendo un reto para la educación. Es necesario realizar investigaciones que examinen esta corriente desde modalidades emergentes, tal como en el caso de la modalidad B-Learning de la UNEFM.

\section{Referencias}

Cánchica, M (2009). Reporte de lectura crítica del libro: Aprendizaje dialógico interactivo, una herramienta para el logro de una educación para la libertad. Trabajo no publicado. Universidad Nacional Politécnica de la Fuerza Armada Nacional (UNEFA), Caracas, Distrito Federal, República Bolivariana de Venezuela.

Cánchica, M. (2014). Innovación Educativa en la Modalidad B-Learning fundamentada en el Aprendizaje Dialógico Interactivo. Tesis Doctoral no publicada. Universidad Nacional Experimental Politécnica de la Fuerza Armada Nacional Bolivariana, Caracas, Distrito Federal, Venezuela.

Coello, Y. (2010). Innovación Curricular en las universidades experimentales de Venezuela. Tesis Doctoral no publicada. Universidad Nacional Experimental Politécnica de la Fuerza Armada Nacional Bolivariana, Caracas, Distrito Federal, Venezuela

Coello, Y. \& Perozo, R (2005). Justificación del cambio de denominación Estudios Dirigidos al de Aprendizaje Dialógico Interactivo. UNEFM -Vicerrectorado Académico. Documento institucional. CoroVenezuela.
Coello, Y.; Hernández, E; \& Subero, O (2002). Proyecto Experimental de Estudios Dirigidos. UNEFMVicerrectorado Académico. Documento institucional. Coro-Venezuela.

Coello, Y.; Peña, K \&, Pernalete, D. (2008). FEDITIC (2009). Programa de Formación Docente en Estrategias Didácticas con tecnologías de Información y Comunicación bajo el Enfoque Dialógico e Interactivo" de la Universidad Nacional Experimental "Francisco de Miranda". Material institucional

Freire, P. (1997). A la sombra de este árbol. Barcelona: El Roure Ciencia.

Freire, P. (2002). Pedagogía del oprimido. 16a edición. Madrid: Siglo XXI de España.

García, L. (2001). La educación a distancia. De la Teoría a la Práctica. Barcelona, Editorial Ariel.

Goetz J P \& Le Compte, (1988). Etnografia y diseño cualitativo de la investigación. Madrid Morata

Pernalete D.; Cánchica; M. \& Díaz, H (2008). Propuesta de una Ontología para la modalidad mixta de Aprendizaje Dialógico Interactivo de la UNEFM. Tercera Conferencia Latinoamericana de Tecnología de Objetos de Aprendizaje. LACLO. 2008. ISBN 978-970-728-067-0. Pag.101, 2008.

Reglamento EDI/ADI. (2002). Artículos de la modalidad B-Learning de la UNEFM. Disponible en: <http:// adi.unefm.edu.ve/mod/resource/view.php? $\mathrm{id}=5>$ [consulta: 05/09/2010]

Strauss, A. \& Corbin, J (2002). Bases de la investigación cualitativa. Técnicas y procedimientos para desarrollar la teoría fundamentada. Editorial Universidad de Antioquia. 
Talavera, J. \& Yépez, Y (2009). Aprendizaje dialógico interactivo. Una herramienta para el logro de una educación para la libertad", autores: Ediciones UNIMETROPA. Venezuela.

Torres, C. I. (2014). El B-Learning: un acercamiento al estado del conocimiento en Iberoamérica, 20032013. Editorial Universidad de Guadalajara. Disponible en: <http://148.202.195.107:8080/jspui/ handle/123456789/55955> [consulta: 25/05/2012]

Turpo, O (2012).La Modalidad Educativa Blended Learning en las Universidades de Iberoamérica: Análisis y Perspectivas de Desarrollo. Revista Educar 2012, vol. 48/1 123-147.

Wagner, E.D. (1994). In support of a functional definition of interaction. The American Journal of Distance Education, 8 (2), 6-29. 